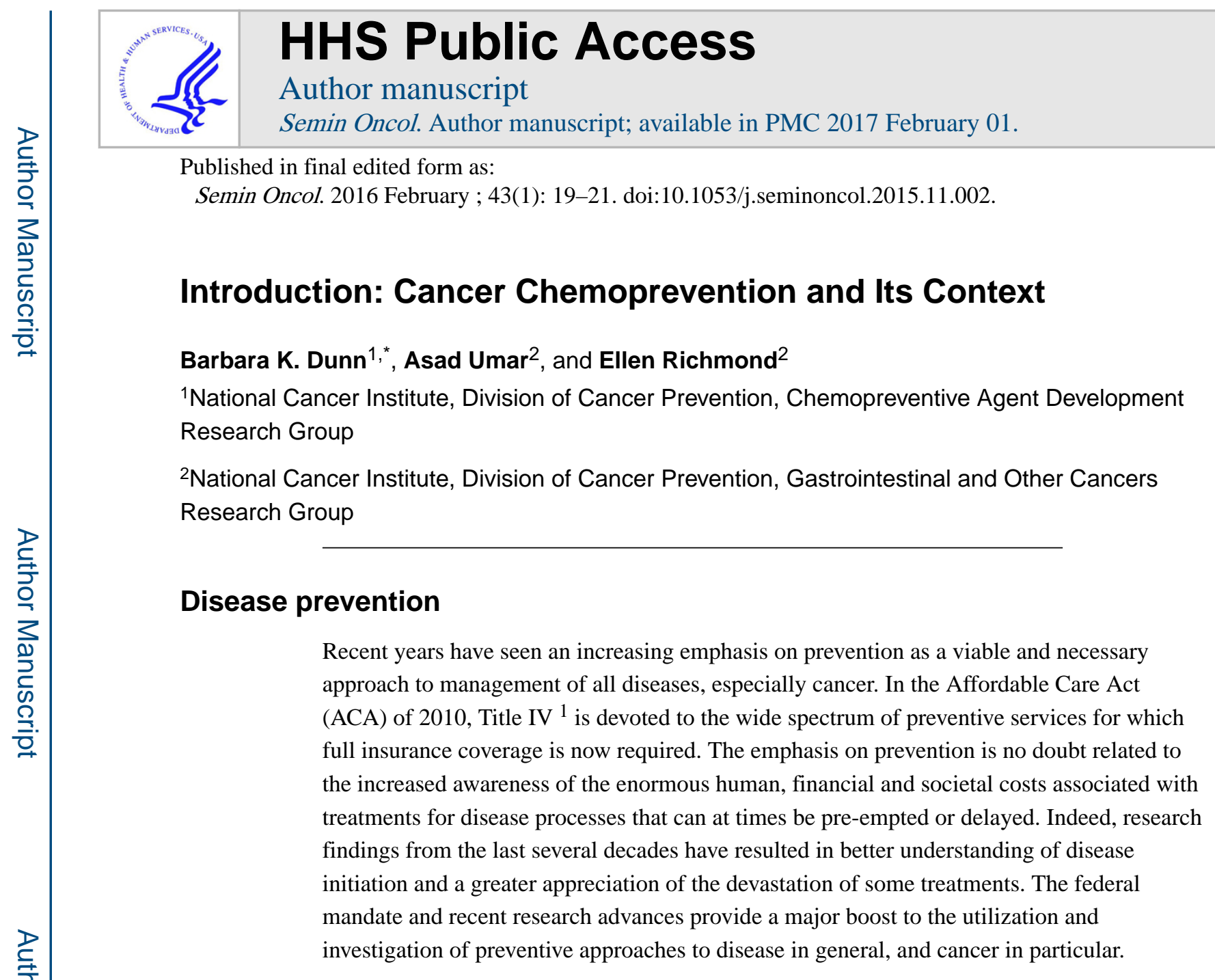

\title{
Cancer prevention
}

Thus, the field of cancer prevention has come to the forefront as the preferred approach to cancer management, with increasing attention being paid to research in this area ${ }^{2}$. The scope of cancer prevention research is broad, as is evident in the review by Lippman and Hawk in $2010^{3}$ and in the two 2010 issues of Seminars in Oncology ${ }^{4-5}$ that are devoted to this research area. At one extreme, observational studies investigate associations between putative (endogenous and exogenous) carcinogenic exposures and cancer risk, while experimental laboratory work explores the underlying molecular mechanisms that imbue such factors with malignant properties. Studies of this nature lay the groundwork for applied research that directly tests effects due to the withdrawal of risk-conferring factors or the introduction of proposed preventive agents. Associations discovered in epidemiologic studies are thus hypothesis-generating, while laboratory findings can be confirmatory of such associations and reveal biomarkers and targets of chemopreventive agents.

Experimental research allows manipulation of carcinogenesis by introduction of either pro-

*Corresponding author: dunnb@mail.nih.gov.

Publisher's Disclaimer: This is a PDF file of an unedited manuscript that has been accepted for publication. As a service to our customers we are providing this early version of the manuscript. The manuscript will undergo copyediting, typesetting, and review of the resulting proof before it is published in its final citable form. Please note that during the production process errors may be discovered which could affect the content, and all legal disclaimers that apply to the journal pertain. 
or anti-carcinogenic molecules. Together these early-stage research approaches serve to generate hypotheses that underlie the translation of suggestive findings into preclinical and then clinical studies and eventually patient care.

Investigating the effects of potential preventive interventions is a central goal in cancer preventive research. Elimination of proven risk-inducing exposures, such as tobacco, offers a type of intervention that can be tested for risk-reducing efficacy in preclinical animal models and eventually in clinical trials. Conversely, active intervention with anti-carcinogenic agents, including drugs and natural products/nutrients, or even lifestyle modifications such as physical activity and diet, can be tested for cancer preventive efficacy in animals as well as in humans in clinical trials. Population-level screening for purposes of early diagnosis of existing cancer can unearth benign, although premalignant, lesions, thereby playing an important role in cancer prevention research. Such lesions identify individuals at increased risk of invasive cancer, placing them within a cohort that is considered appropriate for testing chemopreventive interventions.

The notion that prevention is possible is intricately intertwined with the very nature of carcinogenesis. The common adult epithelial tumors (such as cancers of the breast, prostate, colon, lung, cervix and ovary) develop over many years via a multistep process involving the accumulation of mutations in the cancer cell genomes together with contributory changes in the microenvironment, including the surrounding elements of the immune system ${ }^{3}$. This long latent period, with a generally slow evolution from normal through increasingly aggressive neoplastic stages to frank, invasive cancer presents an opportunity to intervene with preventive modalities ${ }^{6}$. The challenge is to identify individuals who will benefit most from preventive interventions, that is, those who are harboring or at risk of developing such precancerous neoplastic changes in vulnerable tissues.

\section{Chemoprevention: history}

Chemoprevention, the topic of this volume, is best understood within the broad context of cancer prevention. The concept of administering agents with the intent of inhibiting progression to cancer was referred to as "chemoprophylaxis" by Lee Wattenberg in a 1966 review of experimental chemical inhibition of chemically induced carcinogenesis in animals ${ }^{7}$. The actual term "chemoprevention" was coined in 1976 by Sporn et al. ${ }^{8}$ to describe "a new pharmacologic approach to the prevention of cancer...". The chemopreventive intervention prevents, suppresses, or reverses the initiation of carcinogenesis or the progression of already initiated, neoplastic cells to invasive cancer ${ }^{9-11}$. In one interpretation of this approach, noncytotoxic nutrients, or synthetic chemical or biological compounds are introduced to protect against the development and progression of mutant clones of malignant cells ${ }^{11-12}$. A narrower definition of chemoprevention specifically excludes compounds in food consumed in a normal diet ${ }^{13}$. Although specific constituents of some dietary components, such as beta-carotene, calcium, vitamin D, sulforaphane, and resveratrol, may constitute chemopreventive agents, the foods containing these elements fall instead within the purview of "diet and cancer", not "chemoprevention". 


\section{Chemoprevention: this volume}

Outside of the cancer prevention research community, the most visible examples of clinical chemoprevention research are the large phase 3 clinical trials testing agents for their ability to reduce the risks of breast and prostate cancer ${ }^{14-18}$. Demonstration that the chemopreventive agents, tamoxifen and raloxifene, significantly reduced the incidence of breast cancer led to the approval of these agents for this indication by the Food and Drug Administration (FDA) in $1998{ }^{19}$ and $2007^{20}$, respectively. Although showing efficacy in reducing the risk of prostate cancer, finasteride and dutasteride also exhibited toxicity (most notably, an apparent increase in high-grade cancers) that was concerning enough to deter the FDA from granting them similar preventive approval ${ }^{21}$. This exemplifies a central theme that runs through the development of agents for chemoprevention: intolerance of toxicity. Prevention research, because it is directed ultimately toward otherwise healthy individuals who, although at increased risk, may remain cancer-free with no preventive intervention, entails even greater vigilance regarding adverse events than is required in the treatment research setting where trial participants already have cancer. These important examples of chemoprevention have been extensively discussed in earlier reviews ${ }^{22}$. In the present volume, rather than reviewing research for each of the major cancer sites or presenting a comprehensive discussion of agents that are in ongoing prevention studies, we have chosen to select conceptual themes that reflect current, often novel, approaches to chemoprevention research. To a certain extent, this approach reflects the current understanding that molecular processes that drive cancer are not necessarily unique to one organ site. ${ }^{23}$. Given concerns about adverse outcomes, trials using alternative, hopefully less toxic, approaches to administering drugs with documented preventive efficacy are discussed in this volume. One such approach that involves reducing dosage and/or administering agents at lower frequencies, using intermittent dosing, is being applied to tamoxifen for breast cancer prevention. Another effort to ameliorate systemic toxicity involves local application to the organ at risk, as seen with topical gels containing drugs known to decrease risk of breast cancer. Toxicity concerns have also been addressed by recruiting agents with an extensive history of use for non-cancer diseases to cancer prevention applications, commonly referred to as "drug repurposing". Signals of lower cancer incidence can emerge from post-marketing data on widely used agents such as metformin for diabetes. Because their toxicity is well established and is usually acceptable for healthy individuals, repurposing of such approved agents to cancer prevention is becoming increasingly attractive, as presented in this volume.

Whereas the previous Seminars in Oncology: Cancer Prevention I volume contained comprehensive reviews of bioactive food components and their cancer preventive activities ${ }^{24-25}$, the current volume pays tribute to this category of chemopreventive agents by highlighting a single promising and well-studied preventive nutrient, sulforaphane, a component of cruciferous vegetables. The relevance of nutrients is also seen, however, in the comprehensive discussion of retinoids, a class of chemical compounds related to vitamin A that is well known for chemopreventive activity. The importance of nutritional interventions also appears in the paper addressing inflammation and its association with obesity and metabolic syndrome, an area that has emerged as a major focus of cancer research. Omega-3-fatty acids, a group of polyunsaturated fats found in certain plant and fish oils, are 
also under investigation as a chemopreventive strategy that may work through its antiinflammatory activity.

Molecular pathways involved in misdirected metabolism and inflammatory cytokines resulting from immune dysregulation are incorporated into prevention research as markers of cancer predisposition, modulatable markers of chemopreventive efficacy and targets of chemopreventive interventions. An extensive body of literature has documented the cancer preventive properties of nonsteroidal anti-inflammatory drugs (NSAIDs), especially aspirin, as discussed in this issue. The repurposing of metformin from diabetes treatment to cancer preventive intervention, based on epidemiologic signals, gains additional justification on molecular mechanistic grounds. This biguanide targets the cell proliferation-inducing mTOR pathway which is upregulated in tissues exhibiting metabolic abnormalities. Another class of chemopreventive agents that may work through anti-inflammatory mechanisms are the omega-3-fatty acids, mentioned above in the context of nutritional interventions.

Recruitment of the immune system to prevent cancer has a much broader application than merely in relation to inflammation, as discussed in the paper on immunoprevention. Vaccines to prevent cancer, particularly those associated with infectious agents (hepatitis B virus/HBV and human papillomavirus/HPV) are now familiar, HBV vaccines having been in widespread use since the 1980s. More recently vaccine research is targeting tumorassociated antigens, the altered-self antigens found primarily on cancer cells, bringing cancers with no known association with infection into the domain of targeted immunotherapy. In contrast to the adaptive immune responses elicited by vaccines, agents that stimulate the innate component of the immune system modulate immunity in a nonspecific manner to antagonize proliferation of cancer cells. These nonspecific agents are being applied increasingly to the treatment and prevention of cancer.

Also discussed in two entries in this volume is an area of intense interest that is related to modulation of the immune system: the microbiota with their collective genomes, the microbiome. Association studies have documented particular constellations of microbiota in healthy versus compromised tissue at various organ sites. Beyond associations, physiologic investigations suggest that appropriate manipulation of microbiota can lead to reduced risk of cancer.

This volume begins with a foundational piece discussing the molecular and physiologic activities that have been deemed the "hallmarks of cancer" 26-27 and the way in which aberrations in these domains contribute to carcinogenesis. The genetic complexity that underlies these "hallmark" physiologic changes is apparent in all cancers. Adenocarcinoma of the esophagus, and its premalignant precursor, Barrett's esophagus, has been singled out as one example where the clonal evolution of the genetic aberrations that contribute to carcinogenesis has been extensively studied. The genetic heterogeneity observed in lesions of this esophageal cancer subtype is typical of the cell populations in other solid tumors, pointing to a complexity that is now accepted as the norm for cancer ${ }^{28-30}$.

Investigations into the genetic/genomic manifestations of cancer have thus assumed a forefront position in cancer research. Revelation of the genetic landscape of cancers and 
tissue fields at risk of cancer provide the groundwork for developing "targeted" therapies and identifying at-risk individuals. We offer a discussion of modern laboratory technologies used for analysis of large, genomic data. Critical to the interpretation of data generated from these high throughput experimental platforms is the development of companion software platforms to decipher valid associations generated as a result of massive multiple testing. The application of these genetic/genomic/epigenetic/epigenomic discoveries to cancer prevention is addressed. Another paper devoted to the practical application of genetics introduces the notion that rare inherited cancer syndromes, often viewed as esoteric and not immediately relevant to a public health orientation, have a useful role in cancer prevention research. These genetic syndromes offer model systems in which agents can be tested for their ability to prevent cancers for which the affected individuals are at extremely high risk.

In a digression from the usual domain of cancer prevention, we have included a paper on preventing cancers in the context of infection with HIV. HIV-infected individuals have long been known to be at elevated risk of a number of specific cancers and cancer-like syndromes, notably Kaposi's sarcoma, multicentric Castleman disease, primary central nervous system lymphomas, and primary effusion lymphomas. Treatment of cancer in these patients has posed a challenge, given their already immunocompromised state. Now that highly active combined antiretroviral therapy (cART) with multiple agents has enabled the long-term survival of individuals infected with this virus, efforts to prevent such cancers are timely and important.

In this volume of Seminars in Oncology we have selected topics that cover the diverse approaches to chemoprevention research. This area of research has expanded well beyond the administration of conventional pharmaceutical agents and bioactive food components. A discussion of the PREVENT chemopreventive agent development program in the National Cancer Institute's Division of Cancer Prevention illustrates the breadth of pharmaceutical approaches that are being tested in the preclinical setting. In addition to agent development, remaining challenges include identifying appropriate candidates for these interventions, i.e. individuals at high risk of developing cancer and at low risk of agent-induced toxicity. Keeping these stipulations in mind, chemoprevention research is ongoing at all stages, from preclinical testing to clinical trials of potential preventive interventions.

\section{Aims of Seminars in Oncology: Chemoprevention}

Among the aims of this volume is to discuss data from new studies that test specific novel hypotheses that underlie emerging strategies for preventing cancer. Importantly, these discussions are intended to stimulate interest in the field of chemoprevention. In addition, the compilation of prevention topics in a single volume should foster synthesis of the various approaches and the generation of new hypotheses and methods for preventing cancer.

Advancing the field of chemoprevention will require careful communication, adhering to a common standard of terminology. For example, pre-invasive stages of carcinogenesis differ from both normal tissue and from cancer, a distinction that is not always adequately acknowledged. Furthermore, even within the pre-invasive continuum, the range of lesions varies with respect to malignant potential, necessitating adherence to an accepted descriptive 
language. Rigor in applying prevention-specific terminology is critical to the development and exploration of new hypotheses regarding promising chemopreventive interventions.

Throughout the discussions of the diverse research areas covered by the contributing authors, the criticality of understanding carcinogenesis at the genetic and molecular levels is evident. The new technologies allow us to unravel the nuances of carcinogenesis and interfere with its progression in order to reduce the incidence of clinically meaningful cancer.

\section{References}

1. Patient Protection and Affordable Care Act; Public Law No. 111-148, Title IV; 2010 Vol Public Law No. 111-148, Title IV 2010.

2. Blackburn EH. Highlighting the science of cancer prevention. Cancer Prev Res (Phila). Apr.2010 3(4):393. [PubMed: 20354167]

3. Lippman SM, Hawk ET. Cancer prevention: from 1727 to milestones of the past 100 years. Cancer Res. Jul 1; 2009 69(13):5269-5284. [PubMed: 19491253]

4. Dunn, BK.; Greenwald, P. Seminars in Oncology: Cancer Prevention I. New York, NY: Elsevier; 2010.

5. Dunn, BK.; Greenwald, P. Seminars in Oncology: Cancer Prevention II. New York, NY: Elsevier; 2010.

6. Kelloff GJ, Lippman SM, Dannenberg AJ, et al. Progress in chemoprevention drug development: the promise of molecular biomarkers for prevention of intraepithelial neoplasia and cancer--a plan to move forward. Clin Cancer Res. Jun 15; 2006 12(12):3661-3697. [PubMed: 16778094]

7. Wattenberg LW. Chemoprophylaxis of carcinogenesis: a review. Cancer Res. Jul; 1966 26(7):15201526. [PubMed: 5330111]

8. Sporn MB, Dunlop NM, Newton DL, Smith JM. Prevention of chemical carcinogenesis by vitamin A and its synthetic analogs (retinoids). Fed Proc. May 1; 1976 35(6):1332-1338. [PubMed: 770206]

9. Kelloff GJ, Sigman CC, Greenwald P. Cancer chemoprevention: progress and promise. Eur J Cancer. Dec; 1999 35(13):1755-1762. [PubMed: 10673988]

10. Wu X, Patterson S, Hawk E. Chemoprevention--history and general principles. Best Pract Res Clin Gastroenterol. Aug; 2011 25(4-5):445-459. [PubMed: 22122762]

11. William WN Jr, Heymach JV, Kim ES, Lippman SM. Molecular targets for cancer chemoprevention. Nat Rev Drug Discov. Mar; 2009 8(3):213-225. [PubMed: 19247304]

12. Greenwald P, Kelloff G, Burch-Whitman C, Kramer BS. Chemoprevention. CA Cancer J Clin. JanFeb;1995 45(1):31-49. [PubMed: 7804897]

13. Bertram JS, Kolonel LN, Meyskens FL Jr. Rationale and strategies for chemoprevention of cancer in humans. Cancer Res. Jun 1; 1987 47(11):3012-3031. [PubMed: 3105872]

14. Fisher B, Costantino JP, Wickerham DL, et al. Tamoxifen for prevention of breast cancer: report of the National Surgical Adjuvant Breast and Bowel Project P-1 Study. J Natl Cancer Inst. Sep 16; 1998 90(18):1371-1388. http://www.ncbi.nlm.nih.gov/entrez/query.fcgi? cmd=Retrieve \&db=PubMed\&dopt=Citation\&list_uids=9747868. [PubMed: 9747868]

15. Vogel VG, Costantino JP, Wickerham DL, et al. Effects of tamoxifen vs raloxifene on the risk of developing invasive breast cancer and other disease outcomes: the NSABP Study of Tamoxifen and Raloxifene (STAR) P-2 trial. Jama. Jun 21; 2006 295(23):2727-2741. [PubMed: 16754727]

16. Vogel VG, Costantino JP, Wickerham DL, et al. Update of the National Surgical Adjuvant Breast and Bowel Project Study of Tamoxifen and Raloxifene (STAR) P-2 Trial: Preventing breast cancer. Cancer Prev Res (Phila). Jun; 2010 3(6):696-706. [PubMed: 20404000]

17. Thompson IM, Goodman PJ, Tangen CM, et al. The influence of finasteride on the development of prostate cancer. N Engl J Med. Jul 17; 2003 349(3):215-224. http://www.ncbi.nlm.nih.gov/entrez/ query.fcgi?cmd=Retrieve \&db=PubMed\&dopt=Citation\&list_uids=12824459. [PubMed: 12824459] 
18. Andriole GL, Bostwick DG, Brawley OW, et al. Effect of dutasteride on the risk of prostate cancer. N Engl J Med. Apr 1; 2010 362(13):1192-1202. [PubMed: 20357281]

19. FDA. Tamoxifen for Breast Cancer Risk Reduction in High-risk Women. 1998. http:// www.drugs.com/pro/tamoxifen.html

20. FDA. Raloxifene for Breast Cancer Risk Reduction in High-risk Women. 2007. http:// newsroom.lilly.com/ReleaseDetail.cfm?releaseid=2640360

21. Theoret MR, Ning YM, Zhang JJ, Justice R, Keegan P, Pazdur R. The risks and benefits of 5alphareductase inhibitors for prostate-cancer prevention. N Engl J Med. Jul 14; 2011 365(2):97-99. [PubMed: 21675880]

22. Arun B, Dunn BK, Ford LG, Ryan A. Breast cancer prevention trials: large and small trials. Semin Oncol. Aug; 2010 37(4):367-383. [PubMed: 20816507]

23. Munoz J, Swanton C, Kurzrock R. Molecular profiling and the reclassification of cancer: divide and conquer. Am Soc Clin Oncol Educ Book. 2013:127-134. [PubMed: 23714478]

24. Davis CD, Emenaker NJ, Milner JA. Cellular proliferation, apoptosis and angiogenesis: molecular targets for nutritional preemption of cancer. Semin Oncol. Jun; 2010 37(3):243-257. [PubMed: 20709208]

25. Gullett NP, Ruhul Amin AR, Bayraktar S, et al. Cancer prevention with natural compounds. Semin Oncol. Jun; 2010 37(3):258-281. [PubMed: 20709209]

26. Hanahan D, Weinberg RA. The hallmarks of cancer. Cell. Jan 7; 2000 100(1):57-70. [PubMed: 10647931]

27. Hanahan D, Weinberg RA. Hallmarks of cancer: the next generation. Cell. Mar 4; 2011 144(5): 646-674. [PubMed: 21376230]

28. Gerlinger M, Rowan AJ, Horswell S, et al. Intratumor heterogeneity and branched evolution revealed by multiregion sequencing. N Engl J Med. Mar 8; 2012 366(10):883-892. [PubMed: 22397650]

29. Longo DL. Tumor heterogeneity and personalized medicine. N Engl J Med. Mar 8; 2012 366(10): 956-957. [PubMed: 22397658]

30. Aparicio S, Caldas C. The implications of clonal genome evolution for cancer medicine. N Engl J Med. Feb 28; 2013 368(9):842-851. [PubMed: 23445095] 